\title{
Thalamic Atrophy Plays a Crucial Role in the Effect of Asymptomatic Carotid Stenosis on Cognitive Impairment
}

This article was published in the following Dove Press journal:

Clinical Interventions in Aging

\author{
Wen Zhang,' Zhao Qing, ' \\ Yongwei $\mathrm{Hu},{ }^{2}$ Mingran Shao, (D) \\ Jiaming Lu,' Junxia Wang,' Ming \\ Li,' Xin Zhang,' Zuzana \\ Nedelska, ${ }^{3}$ Jakub Hort, (iD ${ }^{3}$ \\ Zhishun Wang, ${ }^{4}$ Tong Qiao, \\ Bing Zhang' \\ 'Department of Radiology, Drum Tower \\ Hospital, The Affiliated Hospital of \\ Nanjing University Medical School, \\ Nanjing, People's Republic of China; \\ ${ }^{2}$ Department of Vascular and \\ Interventional Radiology, Wujin People's \\ Hospital Affiliated to Jiangsu University, \\ Changzhou, People's Republic of China; \\ ${ }^{3}$ Department of Neurology, Memory \\ Clinic, 2nd Faculty of Medicine, Charles \\ University and Motol University Hospital, \\ Prague, Czech Republic; ${ }^{4}$ Columbia \\ University Vagelos College of Physicians \\ and Surgeons, New York, NY, USA; \\ ${ }^{5}$ Department of Vascular Surgery, Drum \\ Tower Hospital, The Affiliated Hospital of \\ Nanjing University Medical School, \\ Nanjing, People's Republic of China
}

Correspondence: Bing Zhang Department of Radiology, Drum Tower Hospital, The Affiliated Hospital of Nanjing University Medical School, No. 321 Zhongshan Road, Nanjing 210008 ,

People's Republic of China

Tel +86 I585 I803070

Email zhangbing_nanjing@nju.edu.cn

Tong Qiao

Department of Vascular Surgery, Drum Tower Hospital, The Affiliated Hospital of Nanjing University Medical School, No.

321 Zhongshan Road, Nanjing 210008 ,

People's Republic of China

Tel +86 I595I985338

Email qiaotongmail@aliyun.com
Purpose: Our objectives were to assess the abnormalities of subcortical nuclei by combining volume and shape analyses and potential association with cognitive impairment.

Patients and Methods: Twenty-nine patients with severe ACS of the unilateral internal carotid artery and 31 controls were enrolled between January 2017 to August 2018. All participants underwent a comprehensive neuropsychological evaluation, blood lipid biochemical measurements, and structural magnetic resonance imaging (MRI) to measure subcortical volumes and sub-regional shape deformations. Basic statistics, correction for multiple comparisons. Seventeen ACS patients underwent carotid endarterectomy (CEA) within one week after baseline measurements, cognitive assessments and MRI scans were repeated 6 months after CEA.

Results: The ACS patients had higher apolipoprotein B/apolipoprotein A1 (ApoB/ApoA1) ratio and worse performance in all cognitive domains than controls. Moreover, the ACS patients showed more profound thalamic atrophy assessed by shape and volume analysis, especially in the medial dorsal thalamus. No significant differences were found in other subcortical nuclei after multiple comparisons correction. At baseline, thalamic atrophy correlated with cognitive impairment and ApoB/ApoA1 ratio. Furthermore, mediation analysis at baseline showed that the association of carotid intima-media thickness with executive functioning was mediated by thalamic volume. After CEA, cognitive improvement and increase in the bilateral medial dorsal thalamic volume were observed.

Conclusion: Our study identified the distinct atrophy of subcortical nuclei and their association with cognition in patients with ACS. Assessments of the thalamus by volumetric and shape analysis may provide an early marker for cerebral ischemia and reperfusion after CEA. Keywords: asymptomatic carotid stenosis, cognitive impairment, magnetic resonance imaging, endarterectomy, thalamus

\section{Introduction}

Asymptomatic carotid stenosis (ACS) is a common finding in the elderly, especially in patients with coronary artery disease or peripheral artery disease. ${ }^{1}$ Patients with ACS often experience subtle cognitive deficits (including attention, memory, processing speed, and executive function) which have received little attention in the clinical setting, but the cognitive impairment may gradually progress, and eventually contribute to subsequent dementia. ${ }^{2}$ Individuals with carotid atherosclerosis have a 2.4-5.0-fold increased risk of dementia compared with those without carotid stenosis. ${ }^{3}$ Current treatments can hardly reverse or delay the progression of dementia once stenosis becomes severe ${ }^{4}$ With the aging 
population growing worldwide, and increasing prevalence of atherosclerosis, it is imperative to find biomarkers for early diagnosis, prognosis, and monitoring of the cognitive decline. Several studies have reported that patients with severe ACS showed cognitive improvement after 3 or 6 months of carotid endarterectomy (CEA).

Brain atrophy is a sensitive marker of cognitive impairment. ${ }^{5}$ Magnetic resonance imaging (MRI) can noninvasively evaluate brain structural changes and the pathophysiological mechanisms of ACS-related cognitive impairment. ${ }^{6}$ Previous studies using structural MRI have identified cortical atrophy in patients with ACS., ${ }^{6,7}$ Similarly, the atrophy of subcortical nuclei including the thalamus, caudate, putamen, pallidum, hippocampus, amygdala, and nucleus accumbens was also reported in patients with Alzheimer's disease dementia. Moreover, in ACS patients, reduced cerebral blood flow ${ }^{8}$ and decreased vascular reactivity ${ }^{9}$ of the basal nuclei (caudate, pallidum and thalamus) were reported. However, it is unknown how and to what extent the ACS affects the subcortical nuclei and the cognitive function; ie, some of the complex nuclei such as the hippocampus and thalamus can be further divided into several distinct subregions. These subregions might be differentially affected by long-term cerebral hypoperfusion that could contribute to cognitive impairment in ACS patients.

Our objectives were to determine whether subcortical nuclei abnormalities are related to cognitive impairment in ACS patients; to identify potential clinical risk factors for cognitive impairment. We investigated subcortical nuclei using volume and shape analysis in 29 consecutive patients with ACS compared to 31 controls who also underwent neuropsychological evaluations. We determined the correlations of the volumes of subcortical nuclei with cognitive measures, the carotid intima-media thickness (CIMT), and blood lipid biochemistry measures. Moreover, in a subset of patients with ACS who underwent CEA, we evaluated changes on MRI and cognitive decline in a follow-up study to aid future studies examining ACS progression and treatments.

\section{Materials and Methods}

\section{Participants}

This study was conducted from January 2017 to August 2018 at the Drum Tower Hospital Affiliated with Nanjing University, Nanjing, China. We recruited patients with severe, unilateral ACS of the extracranial internal carotid artery (ICA; $\geq 70 \%$ by the color Doppler ultrasound; contralateral carotid stenosis degree $<50 \%$ ). For comparison, we also recruited participants without significant $(\geq 50 \%)$ stenosis of the main cerebral arteries as controls. The inclusion criteria were 1) age between 50 and 85 years; 2) righthanded; 3 ) more than 6 years of education; 4) no evidence of acute ischemic stroke, cerebral hemorrhage or subarachnoid hemorrhage; 5) no dementia. The exclusion criteria for all participants were 1) Mini-Mental State Examination (MMSE) score $<26$ (a cutoff score for mild cognitive impairment suggested by a large-population urban and rural area survey in China ${ }^{10}$ ); 2) severe systemic diseases such as congestive heart failure, infections, kidney disease, or malignancy; 3) major neurological or psychiatric disorder and depression; 4) contraindication for MRI such ad metal implants; 5 ) significant stenosis ( $\geq 50 \%)$ of the intracranial artery stenosis and 6) alcohol or substance abuse. Finally, a total of 29 patients with severe ACS and 31 controls were included in the final analysis. The flowchart is provided in Supplementary Figure 1.

The study was approved by the Ethics Committee of the Drum Tower Hospital Affiliated to Nanjing University Medical School. All subjects volunteered to participate in the study and written informed consent was obtained from all participants.

\section{Clinical Information, Biochemical Variables, and Cognitive Assessments}

The medical histories were recorded on the presence of stroke, hypertension, diabetes mellitus, hypercholesterolemia, cardiovascular diseases, and peripheral arterial occlusive disease. Smoking habits and alcohol consumption, years of education, and resting blood pressure were also recorded. Blood was collected for the measurement of the total cholesterol (TC), triglyceride (TG), apolipoprotein A1 (ApoA1), and apolipoprotein B (ApoB) levels.

A battery of neuropsychological assessments was administered to all participants by a trained neuropsychologist, blind to clinical diagnosis, within 7 days of the MRI scan. The assessments included the MMSE, evaluation of working memory (digit span test, forward and backward), processing speed (Trail-Making Test $\mathrm{A}$ and $\mathrm{B}$ ), verbal fluency (Boston naming test and animal naming test) and executive functioning (Stroop color and word test). Hamilton Rating Scale was used to assess anxiety and depression. The scores for the individual cognitive domains on each of the cognitive assessments were converted to normalized z-scores. 


\section{Carotid Artery Color Doppler Ultrasound}

All participants enrolled were examined by the color Doppler ultrasound. The maximum degree of stenosis was determined based on caliper measurements at the narrowest point in the ICA of the residual lumen and diameter of the ICA according to the NASCET criteria (North American Symptomatic Carotid Endarterectomy Trial (NASCET) Steering Committee, 1991): the degree of stenosis $=(1-$ internal carotid artery blood flow narrowest width/normal stenosis distal internal carotid artery diameter) $\times 100 \%$. According to the above formula, the degree of stenosis can be divided into four levels: mild stenosis $(0-49 \%)$, moderate stenosis $(50-69 \%)$, severe stenosis (70-99\%), and complete occlusion. CIMT was defined as the distance between the intimal-luminal and the medial-adventitial inter-faces of the carotid artery wall represented as a double-line density on the ultrasound image. The measurement of CIMT was performed at three segments in the bilateral carotid arteries: at $1 \mathrm{~cm}$ before the bifurcation, bulb, and $1 \mathrm{~cm}$ after the bifurcation. The average value was considered as the final CIMT. Flow velocities in the common carotid artery (CCA) and internal carotid artery (ICA) were also obtained.

\section{MRI Acquisition}

Brain MRIs were acquired using a 3.0T scanner (Achieva TX, Philips, Eindhoven, Netherlands) with an 8-channel head coil. A sagittal, high-resolution three-dimensional T1weighted imaging sequence with $\mathrm{TR} / \mathrm{TE}=9.8 / 4.6 \mathrm{~ms}$, FOV $=256 \mathrm{~mm} \times 256 \mathrm{~mm}$; flip angle $=8^{\circ}$, and slice thickness $=$ $1 \mathrm{~mm}$. Fluid attenuation inversion recovery (FLAIR) images were acquired to evaluate white matter hyperintensity (WMH) using the following parameters: $\mathrm{TR} / \mathrm{TE}=4800$ / $275 \mathrm{~ms}$, inversion time (TI) $=1650 \mathrm{~ms}$, FOV $=250 \mathrm{~mm} \times 250 \mathrm{~mm}$, and slice thickness $=0.56 \mathrm{~mm}$. 3D timeof-flight (TOF) magnetic resonance angiograms (MRA) were acquired to exam intracranial artery stenosis using the following parameters: $3 \mathrm{D}$ fast-field echo acquisition with C-SENSE $=3.5, \mathrm{FOV}=200 \times 200 \times 134 \mathrm{~mm}^{3}, \mathrm{TE} / \mathrm{TR}=3.5 / 22 \mathrm{~ms}$, flip angle $=18^{\circ}$, voxel size $=0.60 \times 0.93 \times 1.00 \mathrm{~mm}^{3}$.

\section{Image Analyses}

Periventricular and deep WMH were visually evaluated by an experienced radiologist using the Fazekas scale. ${ }^{11}$ Quality control of the tissue segmentation was performed by an experienced radiologist blinded to clinical diagnosis.
No participants were excluded because of poor segmentation or image quality.

For the subcortical nuclei volume and shape analysis, each anatomical image was first preprocessed to perform intensity correction and skull stripping using the Statistical Parametric Mapping 12. FSL toolbox (FMRIB, Oxford, $\mathrm{UK})^{12}$ was used to segment gray matter (GM), white matter (WM), cerebrospinal fluid (CSF), and bilateral nuclei (thalamus, caudate, putamen, pallidum, hippocampus, amygdala, and nucleus accumbens). Additionally, for each participant, we computed the intracranial volume (ICV) and volumes of the individual subcortical nucleus. The ICV was used to adjust for individual differences in head size. For each subcortical nucleus, a vertex index was calculated using the FSL vertex analysis script, which was based on the signed vertical distance from the surface mesh of the corresponding structure in the MNI 152 template. The positive index indicates outward deformations (expansions) of the nucleus's surface, and the negative index indicates inward deformations (shrinking, atrophy). Additionally, we computed the degree of deformation value across all participants, solely within the atrophic areas to increase the sensitivity via fewer multiple comparisons.

When between-group differences in the volumes of the subcortical nuclei were detected, the segmentation of the subregions of these nuclei was performed to assess the microstructural changes on the subregional level. The automated segmentation of the thalamus was performed using the publicly available probabilistic atlas of thalamic subregions based on ex vivo brain MRI and histology, included in the Freesurfer algorithm. ${ }^{13}$ The bilateral thalamus was divided into six subregions: anteroventral (AV), lateral posterior (LP), centromedian (CM), medial dorsal (MD), ventral lateral (VL), and pulvinar (PU) (Figure 1B).

\section{Follow-Up Study}

The indication for carotid endarterectomy (CEA) is defined as a degree of stenosis more than $70 \%$ and instability of carotid plaque according to the class IIa recommendation of the guideline on the management of patients with extracranial carotid and vertebral artery disease $^{14}$ Only 17 ACS patients in our cohort underwent CEA intervention within no later than 1 week after baseline measurements (see Supplementary Table S1 for detailed baseline information), while the others chose to continue conservative treatment for various reasons. Eleven of 17 individuals completed the longitudinal 
A
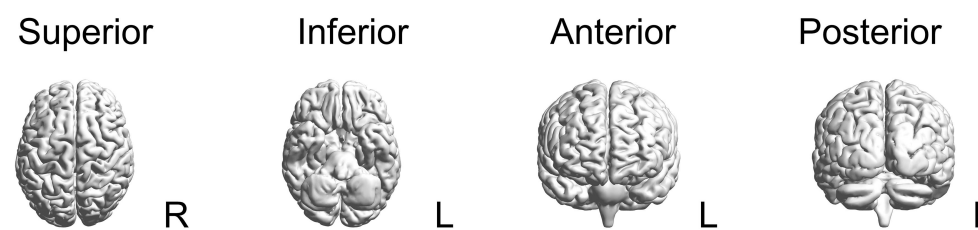

$\mathrm{R}$

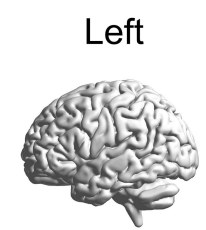

Right
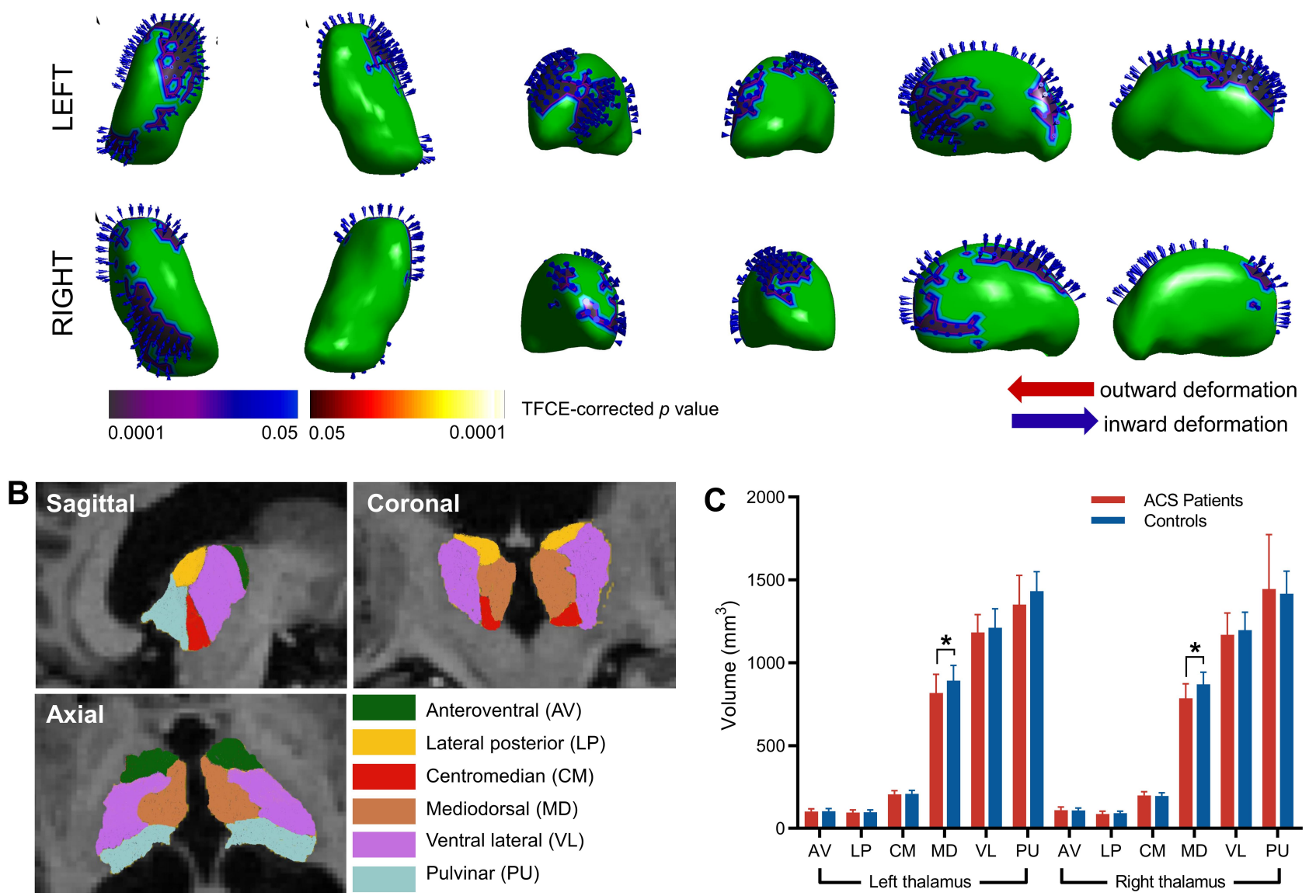

Figure I Subcortical nuclei abnormalities between the ACS patients and controls.

Notes: (A) Vertex-wise shape analysis of the left and right thalamus between ACS patients and normal controls. Blue color or inward arrows indicate regional atrophy. Red color or outward arrows indicate regional expansion. Regional atrophy on the medial-dorsal aspects of the left and right thalamus is shown. TFCE-corrected, $P<0.05$. (B) Thalamus subfields segmentation. 3D TI-weighted images in the sagittal, coronal, and axial planes. (C) The group differences in the thalamic subfields volume at baseline. *Statistically significant after FDR correction.

Abbreviation: ACS, asymptomatic carotid stenosis.

follow-up at 6 months after CEA, unfortunately, another 6 patients refused follow-up or withdrew midway. The same MRI scan and neuropsychology assessment as baseline were performed in follow-up study.

\section{Statistical Analyses}

We used SPSS 23.0 for statistical analyses. The Chi-square test was used to evaluate the categorical variables. The independent sample $t$-test or Mann-Whitney $U$-test was used to compare the continuous variables. In the longitudinal study, the nonparametric Wilcoxon signed-rank test was used to measure the change in thalamic subregional volumes and cognitive decline between baseline and 6 months following the CEA. Significance was considered as a corrected $P<0.05$.

For the volumetric analyses of subcortical nuclei and thalamic subregions, we used a regression-based covariance method to adjust the observed volumes by an amount proportional to the difference between an individual's observed ICV and the mean ICV for all participants. ${ }^{15}$ This method has shown a higher reliability than the proportion method. ${ }^{16}$ The equation for the correction is as follows:

Volume $($ adjusted $)=$ volume $($ observed $)-B\left(I C V_{i}\right.$ $\left.-I C V_{\text {mean }}\right)$, where $\mathrm{ICV}_{\mathrm{i}}=$ the participant's ICV, ICV $\mathrm{Imean}_{\text {m }}$ represents the average ICV, and $\mathrm{B}$ is the slope of the 
regression line between each subcortical nucleus or thalamic subregion $\mathrm{ICV}^{15}$

The group differences in the volume of brain structures and subregions were evaluated using multivariate analysis of covariance (MANCOVA) controlling for age, sex, education, intracranial volume, hypertension, diabetes, coronary heart disease, and smoking. False discovery rate (FDR) correction was conducted per hemisphere to measure potentially significant between-group differences. Cohen's $d$ was calculated to evaluate the effect size, where the effect size can be divided into small (0.2), medium (0.5), and large (0.8). Between-group differences in the shape of the subcortical nuclei were assessed using a general linear model for each nucleus using the FSL randomize procedure. Findings were corrected by the threshold-free cluster enhancement (TFCE) method with 5000 random permutations. ${ }^{17}$

The significant structural abnormalities as indicated by the between-group differences in the volumes or the shape deformations were correlated with lipid/metabolic, cognitive z-scores, and CIMT using Spearman correlations. To assess the associations between thalamic volumes, blood lipid/metabolic measures, CIMT, and cognitive z-scores in the entire cohort, the mediation analysis was performed using the PROCESS SPSS macro toolbox. ${ }^{18}$ The indirect effect in the mediation model was estimated using a bias-corrected bootstrap 95\% CIs, and the number of bootstrap samples was 5000 .

\section{Results}

\section{Participants' and Cognitive}

\section{Characteristics at Baseline}

There were no between-group differences in age, sex, years of education, smoking habits, alcohol consumption, vascular risk factors, Fazekas scores and silent infarction (Table 1). The patients with ACS showed a higher ApoB/ ApoA1 ratios compared with controls. The ACS patients performed worse than controls in terms of global cognition, working memory, processing speed, verbal fluency, and executive function (Table 1).

\section{Volume and Shape Abnormalities of Subcortical Nuclei and Their Subregions in Patients with ACS}

No significant differences were observed in the GM, $\mathrm{WM}$, or CSF volumes between the two groups
Table I Baseline Characteristics and Cognitive Assessment Scores of Subjects with or Without ACS

\begin{tabular}{|c|c|c|c|}
\hline Characteristics & $\begin{array}{l}\text { Controls } \\
(n=31)\end{array}$ & $\begin{array}{l}\text { ACS } \\
\text { Patients } \\
(n=29)\end{array}$ & $P$ value \\
\hline Age, y & $67 \pm 6$ & $68 \pm 8$ & 0.68 \\
\hline Sex, male (\%) & $20(65)$ & $23(80)$ & 0.21 \\
\hline Education, y & $12 \pm 4$ & $10 \pm 3$ & 0.16 \\
\hline Current smoker (\%) & $8(26)$ & $10(34)$ & 0.46 \\
\hline Alcohol consumption (\%) & $9(29)$ & $3(10)$ & 0.07 \\
\hline Hypertension (\%) & $15(48)$ & $19(66)$ & 0.18 \\
\hline Diabetes mellitus (\%) & $15(48)$ & $10(34)$ & 0.28 \\
\hline Dyslipidemia (\%) & $8(26)$ & $10(34)$ & 0.46 \\
\hline Ischemic heart disease (\%) & $3(10)$ & $8(28)$ & 0.07 \\
\hline Peripheral arterial occlusive disease (\%) & $0(0)$ & $3(10)$ & 0.11 \\
\hline Fazekas score & $2.1 \pm 1.2$ & $2.0 \pm 1.6$ & 0.79 \\
\hline Lacunar infarction (\%) & $7(23)$ & $8(28)$ & 0.66 \\
\hline Silent infarction (\%) & $3(10)$ & $4(14)$ & 0.70 \\
\hline \multicolumn{4}{|l|}{ Carotid variables } \\
\hline Main plaque side, left & - & $12(4 \mid)$ & - \\
\hline Degree of stenosis (Doppler), \% & - & $86 \pm 11$ & - \\
\hline $\mathrm{PSV}_{\mathrm{ICA}}, \mathrm{cm} / \mathrm{s}$ & $95 \pm 16$ & $415 \pm 72$ & $<0.001$ \\
\hline $\mathrm{EDV}_{\mathrm{ICA}}, \mathrm{cm} / \mathrm{s}$ & $42 \pm 4$ & $91 \pm 18$ & $<0.001$ \\
\hline $\mathrm{PSV}_{\mathrm{ICA}} / \mathrm{PSV} \mathrm{V}_{\mathrm{CCA}}$ & $2 \pm 1$ & $7 \pm 2$ & $<0.001$ \\
\hline CIMT, mm & $0.6 \pm 0.1$ & $1.2 \pm 0.1$ & $<0.001$ \\
\hline \multicolumn{4}{|l|}{ Clinical variables } \\
\hline Systolic BP, mmHg & $129 \pm 15$ & $136 \pm 17$ & 0.08 \\
\hline Diastolic BP, mmHg & $80 \pm 15$ & $72 \pm 12$ & 0.06 \\
\hline $\mathrm{TG}, \mathrm{mmol} / \mathrm{L}$ & $1.2 \pm 0.8$ & $1.4 \pm 0.5$ & 0.03 \\
\hline $\mathrm{TC}, \mathrm{mmol} / \mathrm{L}$ & $3.9 \pm 1.0$ & $4.1 \pm 1.3$ & 0.49 \\
\hline ApoB/ApoAl & $0.7 \pm 0.2$ & $1.0 \pm 0.3$ & $<0.001$ \\
\hline \multicolumn{4}{|l|}{ Cognitive assessment } \\
\hline Mini-Mental State Examination & $29 \pm 1$ & $28 \pm 1$ & 0.006 \\
\hline \multicolumn{4}{|l|}{ Working memory } \\
\hline DST, forward & $9 \pm 1$ & $7 \pm 1$ & $<0.001$ \\
\hline DST, backward & $6 \pm 1$ & $4 \pm 1$ & $<0.001$ \\
\hline \multicolumn{4}{|l|}{ Processing time } \\
\hline TMT-A & $26 \pm 10$ & $52 \pm 12$ & $<0.001$ \\
\hline TMT-B & $130 \pm 50$ & $159 \pm 48$ & 0.003 \\
\hline \multicolumn{4}{|l|}{ Verbal fluency } \\
\hline ANT & $18 \pm 5$ & $13 \pm 5$ & $<0.001$ \\
\hline BNT & $56 \pm 4$ & $47 \pm 8$ & $<0.001$ \\
\hline \multicolumn{4}{|l|}{ Executive time } \\
\hline Stroop-color & $20 \pm 7$ & $22 \pm 6$ & 0.10 \\
\hline Stroop-word & $24 \pm 8$ & $30 \pm 9$ & 0.003 \\
\hline Stroop-color word & $33 \pm 11$ & $53 \pm 15$ & $<0.001$ \\
\hline
\end{tabular}

Note: Values are presented as the mean \pm SD or $\mathrm{n}(\%)$.

Abbreviations: ACS, asymptomatic carotid stenosis; BP, blood pressure; TG, triglyceride; TC, total cholesterol; ApoB, apolipoprotein B; ApoAI, apolipoprotein AI; PSV, peak systolic velocity; EDV, end-diastolic velocity; CCA, common carotid artery; ICA, internal carotid artery; CIMT, carotid intima-media thickness; DST, digit span test; TMT, trail-making test; ANT, animal naming test; BNT, Boston naming test. 
Table 2 Global Measurements and Subcortical Nuclei Volumes in ACS Patients and Controls at Baseline

\begin{tabular}{|c|c|c|c|c|c|}
\hline \multirow[t]{2}{*}{ Volume, $\mathrm{cm}^{3}$} & \multirow[t]{2}{*}{ Controls $(n=3 I)$} & \multirow[t]{2}{*}{ ACS Patients $(n=29)$} & \multicolumn{2}{|c|}{ Significance } & \multirow{2}{*}{$\begin{array}{l}\text { Effect Size } \\
\text { Cohen's d }\end{array}$} \\
\hline & & & $\mathbf{F}$ & $P$ & \\
\hline \multicolumn{6}{|l|}{ Global } \\
\hline Intracranial volume & $1482 \pm 177$ & $1434 \pm 140$ & 4.555 & 0.037 & 0.479 \\
\hline Gray matter & $569 \pm 73$ & $538 \pm 68$ & 0.798 & 0.376 & 0.468 \\
\hline White matter & $519 \pm 72$ & $493 \pm 63$ & 0.521 & 0.474 & 0.407 \\
\hline Cerebrospinal fluid & $588 \pm 7 \mid$ & $561 \pm 75$ & 0.119 & 0.732 & 0.421 \\
\hline \multicolumn{6}{|l|}{ Subcortical } \\
\hline \multicolumn{6}{|l|}{ Thalamus } \\
\hline Left & $6.5 \pm 0.7$ & $6.0 \pm 0.7$ & 7.412 & $0.009 *$ & 0.652 \\
\hline Right & $6.3 \pm 0.7$ & $5.7 \pm 0.8$ & 7.826 & $0.007^{*}$ & 0.699 \\
\hline \multicolumn{6}{|l|}{ Caudate } \\
\hline Left & $3.2 \pm 0.4$ & $3.0 \pm 0.4$ & 6.398 & 0.015 & 0.607 \\
\hline Right & $3.3 \pm 0.4$ & $3.0 \pm 0.6$ & 5.078 & 0.029 & 0.440 \\
\hline \multicolumn{6}{|l|}{ Putamen } \\
\hline Left & $4.5 \pm 0.9$ & $4.2 \pm 0.5$ & 4.088 & 0.049 & 0.442 \\
\hline Right & $4.6 \pm 0.7$ & $4.1 \pm 1.0$ & 5.044 & 0.029 & 0.426 \\
\hline \multicolumn{6}{|l|}{ Pallidum } \\
\hline Left & $1.6 \pm 0.5$ & $1.4 \pm 0.4$ & 1.390 & 0.244 & 0.289 \\
\hline Right & $1.6 \pm 0.4$ & $1.4 \pm 0.4$ & 2.668 & 0.109 & 0.421 \\
\hline \multicolumn{6}{|l|}{ Hippocampus } \\
\hline Left & $3.7 \pm 0.4$ & $3.6 \pm 0.6$ & 2.563 & 0.116 & 0.290 \\
\hline Right & $4.1 \pm 0.5$ & $3.8 \pm 0.6$ & 5.753 & 0.020 & 0.544 \\
\hline \multicolumn{6}{|l|}{ Amygdala } \\
\hline Left & $0.8 \pm 0.3$ & $0.8 \pm 0.3$ & 0.009 & 0.927 & -0.077 \\
\hline Right & $1.2 \pm 0.3$ & $1.0 \pm 0.3$ & 0.239 & 0.627 & 0.266 \\
\hline \multicolumn{6}{|l|}{ Nucleus Accumbens } \\
\hline Left & $0.5 \pm 0.1$ & $0.4 \pm 0.1$ & 0.463 & 0.499 & 0.250 \\
\hline Right & $0.3 \pm 0.1$ & $0.3 \pm 0.1$ & 0.995 & 0.323 & 0.137 \\
\hline
\end{tabular}

Notes: Adjusted for age, sex, education, intracranial volume, hypertension, diabetes, coronary heart disease, and smoking. *Statistically significant after FDR correction. Abbreviation: ACS, asymptomatic carotid stenosis.

(Table 2). In MANCOVA, we found that volumes in the bilateral thalamus were decreased in the ACS patients (left: $\mathrm{F}=7.412, \mathrm{P}=0.009$; right: $\mathrm{F}=7.826, \mathrm{P}=0.007$ ) after FDR correction. We also observed reduced volume of the bilateral caudate (left: $\mathrm{F}=6.398, \mathrm{P}=0.015$; right: $\mathrm{F}=5.078, \mathrm{P}=0.029$ ), bilateral putamen (left: $\mathrm{F}=4.088$, $\mathrm{P}=0.049$; right: $\mathrm{F}=5.044, \mathrm{P}=0.029)$ and right hippocampus $(\mathrm{F}=5.753, \mathrm{P}=0.02)$; however, these results did not survive the multiple comparison correction (Table 2). Correlation analyses showed that the thalamic volumes were inversely associated with the ApoB/ApoA1 ratios (left thalamus: $r=-0.596, P=0.001$; right thalamus: $r=$ $-0.637, P<0.001)$. The thalamic volume inversely correlated with processing time z-score (left: $r=-0.463$, $P=0.012$; right: $r=-0.598, P=0.001)$ and executive time z-score (left: $r=-0.512, P=0.004$; right: $r=-0.561, P=$ 0.002) (Table 3).

In the shape analysis, we found a deformation (suggesting the atrophy or shrinking) in the anterior medial part of the right and left thalamus in the ACS patients after multiple comparisons correction (all corrected $P<0.05$, TFCE-corrected; left: $P=0.001$; right: $P=0.015$; Figure 1A). No significant outward shape deformations (expansion) were found. The correlation analyses showed that the between-group differences in the shape of the bilateral thalamus were inversely correlated with the ApoB/ 
Table 3 Correlations of Morphometric Abnormalities with Clinical Variables, Cognitive Scores, and Carotid Variables in ACS Patients and Controls

\begin{tabular}{|c|c|c|c|c|c|c|c|c|c|c|c|c|c|}
\hline \multirow[t]{3}{*}{ Group } & \multirow[t]{3}{*}{ Index } & \multicolumn{4}{|c|}{ Subcortical Nuclei Volume } & \multicolumn{4}{|c|}{$\begin{array}{l}\text { Subcortical Nuclei Shape } \\
\text { Deformation }\end{array}$} & \multicolumn{4}{|c|}{ Thalamic Subregion Volume } \\
\hline & & \multicolumn{2}{|c|}{ L_Thalamus } & \multicolumn{2}{|c|}{ R_Thalamus } & \multicolumn{2}{|c|}{ L_Thalamus } & \multicolumn{2}{|c|}{ R_Thalamus } & \multicolumn{2}{|c|}{ L_Mediodorsal } & \multicolumn{2}{|c|}{ R_Mediodorsal } \\
\hline & & $\mathbf{r}$ & $P$ & $\mathbf{r}$ & $P$ & $\mathbf{r}$ & $P$ & $\mathbf{r}$ & $\boldsymbol{P}$ & $\mathbf{r}$ & $\boldsymbol{P}$ & $\mathbf{r}$ & $P$ \\
\hline ACS & ApoB/ApoAI & -0.596 & 0.001 & -0.637 & $<0.001$ & -0.402 & 0.031 & -0.545 & 0.002 & -0.410 & 0.027 & -0.529 & 0.003 \\
\hline \multirow[t]{5}{*}{ Patients } & Working memory & 0.032 & 0.871 & 0.209 & 0.276 & -0.086 & 0.657 & -0.045 & 0.817 & -0.110 & 0.570 & 0.068 & 0.728 \\
\hline & Processing time & -0.463 & 0.012 & -0.598 & 0.001 & -0.403 & 0.030 & -0.460 & 0.012 & -0.371 & 0.047 & -0.304 & 0.109 \\
\hline & Verbal fluency & 0.162 & 0.402 & 0.335 & 0.075 & 0.285 & 0.134 & 0.267 & 0.161 & 0.224 & 0.242 & 0.405 & 0.029 \\
\hline & Executive time & -0.474 & 0.009 & -0.539 & 0.003 & -0.312 & 0.100 & -0.359 & 0.056 & -0.151 & 0.434 & -0.449 & 0.014 \\
\hline & CIMT & -0.512 & 0.004 & -0.561 & 0.002 & -0.390 & 0.037 & -0.430 & 0.020 & -0.468 & 0.010 & $-0.45 I$ & 0.014 \\
\hline \multirow[t]{6}{*}{ Controls } & ApoB/ApoAl & 0.005 & 0.980 & 0.021 & 0.911 & -0.036 & 0.845 & -0.088 & 0.637 & -0.059 & 0.752 & 0 & 0.999 \\
\hline & Working memory & 0.045 & 0.810 & 0.097 & 0.603 & -0.073 & 0.696 & -0.233 & 0.207 & -0.233 & 0.208 & 0.070 & 0.709 \\
\hline & Processing time & 0.182 & 0.327 & 0.116 & 0.535 & -0.205 & 0.268 & 0.044 & 0.814 & 0.277 & 0.131 & 0.121 & 0.518 \\
\hline & Verbal fluency & -0.096 & 0.607 & -0.024 & 0.898 & -0.415 & 0.020 & -0.324 & 0.076 & -0.287 & 0.118 & -0.061 & 0.746 \\
\hline & Executive time & 0.038 & $0.84 I$ & -0.024 & 0.897 & 0.118 & 0.527 & 0.239 & 0.196 & 0.303 & 0.098 & -0.034 & 0.856 \\
\hline & CIMT & -0.274 & 0.136 & -0.213 & 0.249 & 0.100 & 0.593 & -0.041 & 0.827 & 0.075 & 0.69 & 0.112 & 0.549 \\
\hline
\end{tabular}

Notes: Spearman correlation analyses. Compound scores for each cognitive domain were calculated by averaging the z-scores of all individuals.

Abbreviations: L, left; R, right; CIMT, carotid intima-media thickness; ApoB/ApoAI, apolipoprotein B/apolipoprotein AI ratio; ACS, asymptomatic carotid stenosis.

ApoA1 ratio (left: $r=-0.402, P=0.031$; right: $r=-0.545$, $P=0.002$ ). There was a significant inverse correlation between the thalamic shape deformation and processing time z-score (left: $r=-0.403, P=0.03$; right: $r=-0.460$, $P=0.012$ ) (Table 3).

Considering the laterality of carotid stenosis, we did the correlation analysis between CIMT with the volume and shape of ipsilateral and contralateral subcortical nuclei. Then, we found that both thalamic volume and shape on the ipsilateral and contralateral side were significantly correlated with CIMT (all $\mathrm{P}<0.05)$, and the correlation coefficient of ipsilateral side was higher than contralateral side (Supplementary Table S4).

\section{The Volume of Thalamic Subregions}

Figure $1 \mathrm{C}$ and Table 4 show that the volumes of the medial dorsal thalamus (left $\mathrm{MD}: \mathrm{F}=7.707, \mathrm{P}=0.008$; right $\mathrm{MD}: \mathrm{F}=10.678, \mathrm{P}=0.002$ ) were lower in the ACS patients compared with controls. There was an inverse correlation between the medial dorsal thalamic nuclei volumes and the ApoB/ApoA1 ratio (left $\mathrm{MD}: r=$ $-0.410, P=0.027$; right $\mathrm{MD}: r=-0.529, P=0.003)$. The right $\mathrm{MD}$ volume correlated with verbal fluency $\mathrm{z}$-score $(r=0.405, P=0.029)$, and inversely correlated with executive time $z$-score $(r=-0.449, \quad P=0.014)$ (Table 3).

\section{Association Among Carotid Stenosis,} Thalamic Volumes, Clinical Variables, and Cognition

Mediation analysis was applied to test our hypothesis that the reduction of thalamic volumes or clinical and blood biochemistry abnormalities serves as a mediator between the ACS severity and cognitive impairment in ACS patients. Figure 2A demonstrates that the association between CIMT and executive time z-score was in part mediated by the (reduced) left thalamus volume $[\beta$ $=1.8017,95 \%$ CI $(0.3730,4.1001)]$ and (reduced) right thalamus volume $[\beta=2.0893,95 \%$ CI $(0.5056,4.6147)]$. The association between $\mathrm{ApoB} / \mathrm{ApoA} 1$ ratio and right thalamus volume was in part mediated by the CIMT $[\beta=$ $-0.5590,95 \%$ CI $(-1.2762,-0.0377)]$ (Figure 2B).

\section{Effect of Carotid Reperfusion Surgery on Cognitive Function and Thalamic Subregional Volumes}

In the eleven ACS patients with CEA surgery and longitudinal follow up, we observed significant improvement in processing time $z$-score $(p<0.001)$ and executive time z-score $(p=0.006)$ after statistical correction (Figure 3A; Supplementary Table S2), although the cognitive performance remained lower than that of controls. Interestingly, at follow up, we found a significant increase in the 
Table 4 Volumes of Thalamic Subregions in ACS Patients and Controls at Baseline

\begin{tabular}{|c|c|c|c|c|c|}
\hline \multirow[t]{2}{*}{ Volume, $\mathrm{mm}^{3}$} & \multirow[t]{2}{*}{ Controls $(n=3 I)$} & \multirow[t]{2}{*}{ ACS Patients $(n=29)$} & \multicolumn{2}{|c|}{ Significance } & \multirow{2}{*}{$\begin{array}{l}\text { Effect Size } \\
\text { Cohen's d }\end{array}$} \\
\hline & & & $\mathbf{F}$ & $P$ & \\
\hline \multicolumn{6}{|l|}{ Anteroventral (AV) } \\
\hline Left & $104 \pm 16$ & $103 \pm 15$ & 0.009 & 0.923 & 0.048 \\
\hline Right & $109 \pm 15$ & $111 \pm 19$ & 0.112 & 0.739 & -0.182 \\
\hline \multicolumn{6}{|l|}{ Later posterior (LP) } \\
\hline Left & $98 \pm 15$ & $97 \pm 16$ & 0.056 & 0.814 & -0.012 \\
\hline Right & $91 \pm 13$ & $87 \pm 17$ & 0.046 & 0.831 & 0.127 \\
\hline \multicolumn{6}{|l|}{ Centromedian (CM) } \\
\hline Left & $209 \pm 20$ & $205 \pm 22$ & 4.264 & 0.044 & 0.186 \\
\hline Right & $196 \pm 19$ & $199 \pm 23$ & 0.230 & 0.634 & -0.233 \\
\hline \multicolumn{6}{|l|}{ Mediodorsal (MD) } \\
\hline Left & $892 \pm 92$ & $818 \pm 113$ & 7.707 & $0.008^{*}$ & 0.708 \\
\hline Right & $870 \pm 73$ & $799 \pm 103$ & 10.678 & $0.002 *$ & 0.755 \\
\hline \multicolumn{6}{|l|}{ Ventral lateral (VL) } \\
\hline Left & $1211 \pm 1 \mid 4$ & $1183 \pm 107$ & 4.380 & 0.041 & 0.289 \\
\hline Right & $1197 \pm 108$ & $1169 \pm 132$ & 1.249 & 0.269 & 0.114 \\
\hline \multicolumn{6}{|l|}{ Pulvinar (PU) } \\
\hline Left & $1433 \pm 117$ & $1350 \pm 178$ & 5.294 & 0.026 & 0.491 \\
\hline Right & $1416 \pm 136$ & $1444 \pm 329$ & 0.626 & 0.432 & -0.228 \\
\hline
\end{tabular}

Notes: Adjusted for age, sex, education, intracranial volume, hypertension, diabetes, coronary heart disease, and smoking. *Statistically significant after FDR correction. Abbreviation: ACS, asymptomatic carotid stenosis.

bilateral medial dorsal thalamus volume (left: $P=0.045$; right: $P=0.027$; Figure $3 \mathrm{~B}$ ) in CEA patients compared with baseline. However, there was no difference in the whole thalamus volume between the baseline and followup (left: $P=0.120$; right: $P=0.777$; $\underline{\text { Supplementary }}$ Table S3).

\section{Discussion}

In this study using shape and volumetric analyses, we showed the global and local structural abnormalities in the subcortical nuclei are associated with cognitive impairment in ACS patients. ACS patients showed poorer performance compared to controls without ACS in cognitive scores on working memory, processing speed, verbal fluency, and executive functioning. The ACS patients showed atrophy in the medial and dorsal part of the left and right thalamus by volumetric and shape analyses compared to controls. Although the volumes of other subcortical nuclei were also decreased, such as the caudate, putamen and hippocampus, these differences were not statistically significant after statistical correction. Furthermore, the left and right thalamic volume were the mediating factors (mediators) between carotid stenosis and impairment in the executive functioning. Six months after CEA, improvement in cognitive scores and increased bilateral medial dorsal thalamic volume were observed.

Our major finding was that patients with ACS had significantly reduced bilateral thalamic volume, particularly in the medial dorsal thalamus. Previous studies have reported global gray matter atrophy in patients with ACS, ${ }^{7}$ however, these studies did not investigate (or only marginally investigated) the relationship between subcortical nuclei and cognitive impairment. Consistent with previous, ${ }^{19,20}$ the ACS patients in our cohort had a poorer cognitive performance in multiple cognitive domains compared to controls. Additionally, we found thalamic atrophy and medial dorsal thalamic shape deformations (contractions) in the ACS patients, and these morphometric abnormalities correlated with CIMT. The thalamus serves as a pivotal relay station to the cerebral cortex, ${ }^{21}$ and is predominantly supplied with blood by the ipsilateral ICA and basilar artery. ${ }^{8}$ Previous studies have demonstrated impaired cerebral hemodynamic autoregulation in patients with unilateral carotid stenosis both in the ipsilateral and contralateral hemispheres, with more severe in the stenotic side. ${ }^{9}$ Our results also provided evidence 

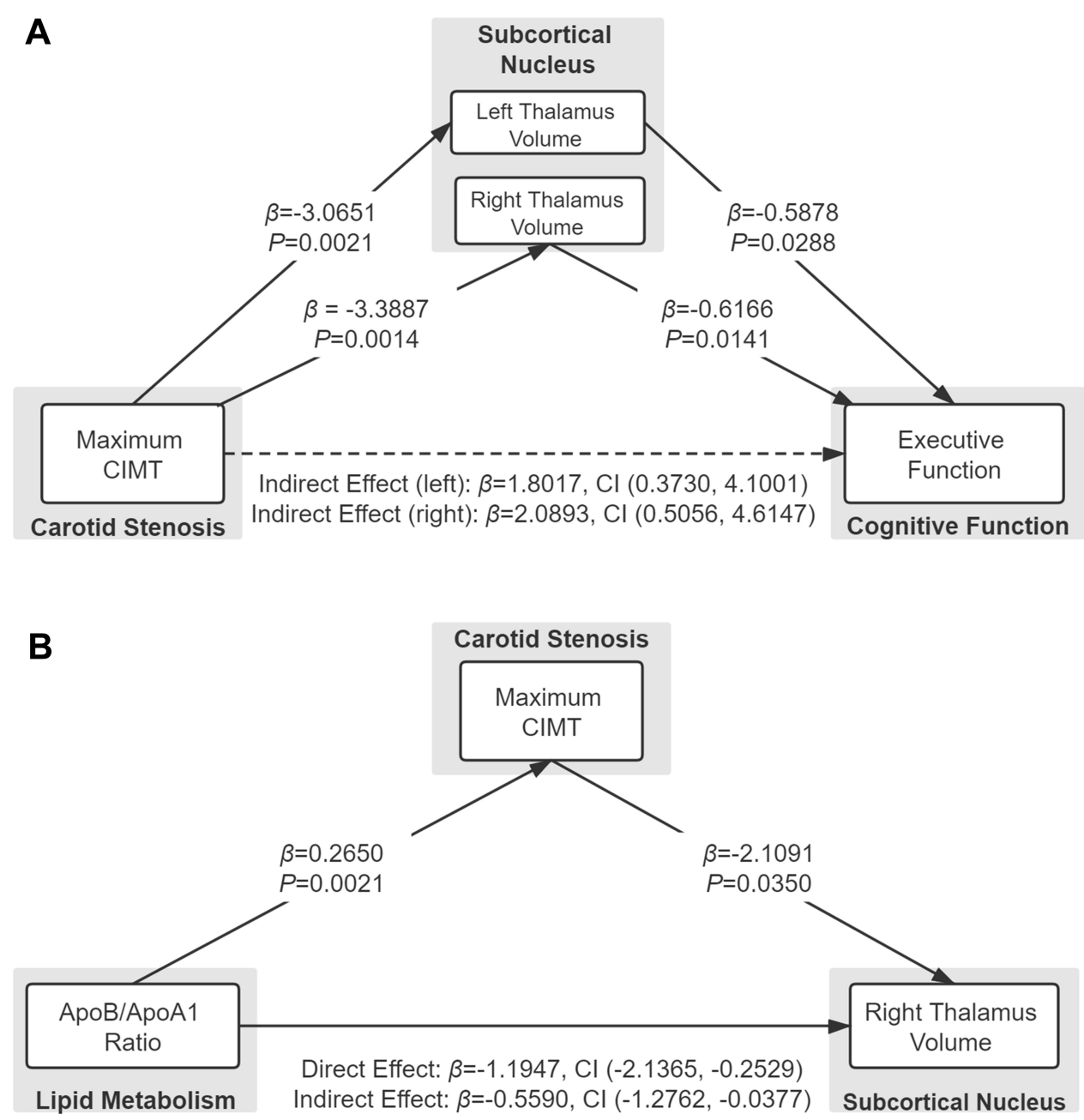

Figure 2 Mediation analysis for associations among carotid stenosis, lipid/metabolic variables, thalamic volume, and cognition at baseline.

Notes: The standardized $\beta$ coefficients were derived from the mediation models. Values are standardized path coefficients with $95 \%$ Cis in parentheses. (A) Association of CIMT, thalamic volume, and executive function (time). (B) Association of ApoB/ApoAI ratio, CIMT and right thalamus volume.

Abbreviations: CIMT, carotid intima-media thickness; ApoB/ApoAI, apolipoprotein B/apolipoprotein AI ratio.

from the side that the stenotic laterality affect both hemisphere differently. Moreover, the medial-dorsal part of the thalamus is anatomically connected to the prefrontal cortex and medial temporal lobes. ${ }^{22}$ Functionally, the medial dorsal thalamus plays an important role in multiple cognitive processes, particularly in executive functioning, associative learning and decision-making. ${ }^{23}$ In our study, we found a significant association between poorer executive function and severe atrophy in the right medial dorsal thalamus, suggesting that atrophy in medial-dorsal thalamus is associated with the impairment in executive function in ACS patients.

The bilateral caudate, putamen and right hippocampus also showed some degree of atrophy, although did not survive the correction. It has been reported that in patients with carotid stenosis, the basal ganglia (caudate, putamen and pallidum) is supplied with blood by the contralateral ICA more often than in normal side, ${ }^{8}$ which might be a compensation strategy to mitigate chronic ischemic damage on the stenotic side. Hippocampal atrophy has been reported in aging-related disease, thus may imply that the mechanism of ACS-related cognitive impairment has some common pathophysiology of aging.

Additionally, we observed that the ApoB/ApoA1 ratio was negatively associated with bilateral thalamic volumes and shape deformations: a higher ApoB/ApoA1 ratio indicated more severe atrophy in the thalamus. The ApoA1 level is a determinant of cholesterol efflux capacity, ${ }^{24}$ and ApoB mainly reflects low-density lipoprotein cholesterol (LDL-C) levels, while increased LDL-C is a major risk factor for the development and progression of atherosclerosis. ${ }^{25}$ On the other hand, 

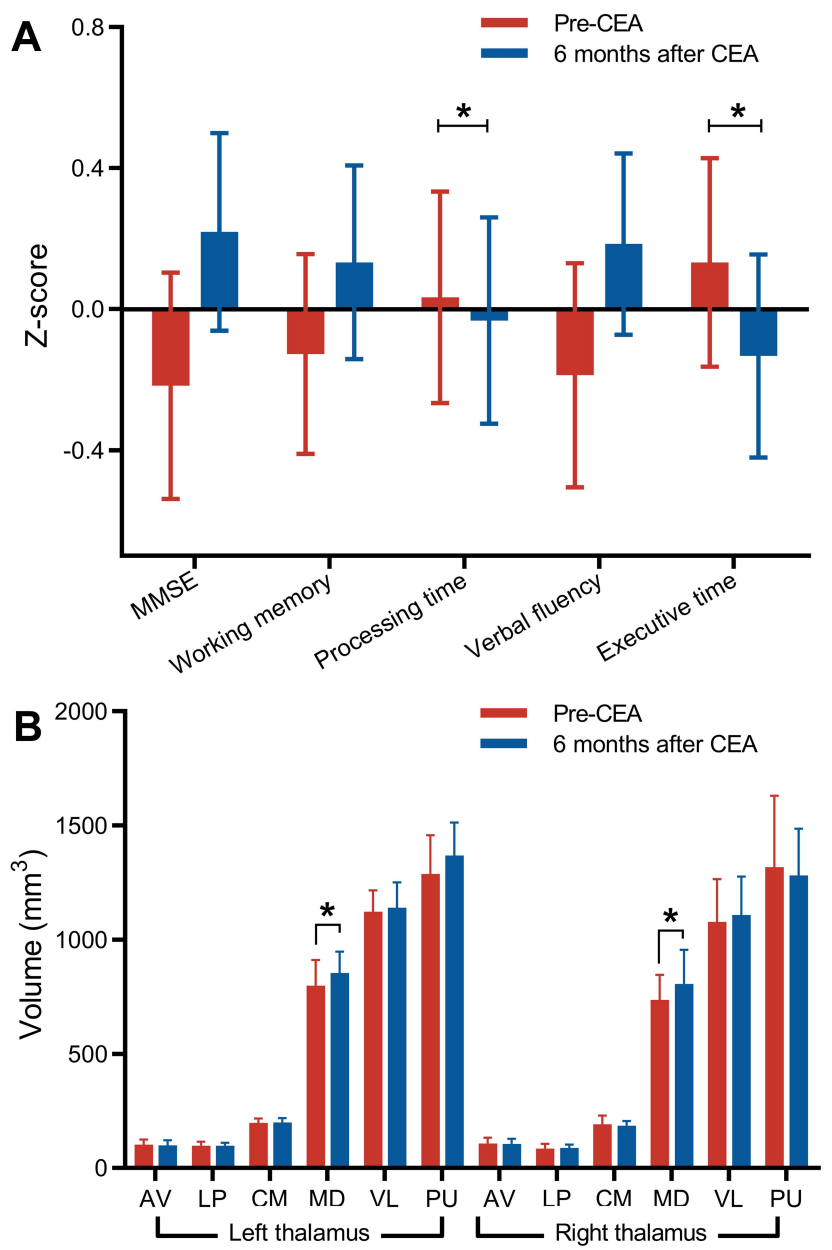

Figure 3 The changes in cognitive assessment scores and thalamic subfield volumes in ACS patients 6 months after CEA.

Notes: (A) Cognitive scores of processing speed and executive function significantly improved 6 months after CEA; ${ }^{*} P<0.01$, Bonferroni corrected. The error bars represent the standard errors of the mean. (B) Changes in the left and right thalamic subfield volumes after CEA; $* P<0.05$.

Abbreviations: CEA, carotid endarterectomy; ACS, asymptomatic carotid stenosis; MMSE, Mini-Mental State Examination; AV, anteroventral; LP, later posterior; $\mathrm{CM}$, centromedian; MD, mediodorsal; VL, ventral lateral; PU, pulvinar.

dyslipidemia, oxidative stress, and inflammation result in endothelial dysfunction, ${ }^{26}$ atherosclerotic plaque formation, and vascular remodeling. Medial thickening and intimal proliferation lead to reduction of luminal diameter, which in turn causes increased resistance to blood flow, decreased cerebral perfusion, and cerebral structural changes. Thus, intensive lipid metabolism control has been widely recommended in the treatment and prevention of carotid stenosis. It was speculated that the thalamus with its multiple reciprocal connections is sensitive to inflammatory processes occurring in the brain. ${ }^{27}$ As a result, the inadequate supply of oxygenrich blood and oxidative stress may injure neurons and later cause thalamic atrophy.
Our mediation model suggested that the bilateral thalamic volume was the mediating factors between CIMT and executive function, and the CIMT was the mediating factors between $\mathrm{ApoB} / \mathrm{ApoA} 1$ ratio and thalamic volume. This may contribute to disentangling the complex associations among atherosclerosis, lipid metabolic disorders, structural brain changes, and cognition. Therefore, early reperfusion and lipid metabolism management may directly or indirectly protect neurons and cognition in patients with $\mathrm{ACS}^{28}$ In our longitudinal study, eleven ACS patients accepted CEA for carotid revascularization and completed follow-up MRI scan and cognitive assessment. Although the effect of CEA on the cognition remains controversial ${ }^{29}$ we found subtle but significant improvement in processing speed, executive functioning, and increased bilateral medial dorsal thalamic volume in follow-up 6 months after baseline. These beneficial changes may be attributed to recovered perfusion and thus potentially enhanced neuronal activity. Indeed, cognitive improvement after CEA in our study are a result of not only the surgical intervention but also the intensive medical management (such as hypertension management, lipid-lowering, and glycemic control). Previous studies have suggested that uncertainty during the CEA can also affect cognitive benefit, including cerebral ischemia during clamping, emboli from the air or the surgical site, oxidative stress, and postoperative cerebral hyperperfusion. ${ }^{30,31}$ Thus, the benefits of surgery should be weighed against short- and long-term complications, but regardless, intensive medical management is of particular importance.

This study had several limitations. First, because males generally have more risks of carotid stenosis than females $^{32}$ and education level can affect cognitive performance, ${ }^{4}$ the difference in the percentage of males $(P=0.21)$ and education levels $(P=0.16)$ between the two groups, though not significant, might have introduced a potential bias. Hence, sex and education were corrected for in our analyses. Second, the sample size was relatively small since ACS is usually an incidental diagnosis. The impact of CEA on cognition and brain structural changes needs to be further investigated in a larger population (contain normal controls, CEA group, and non-CEA group) and over a longer observation period to determine whether thalamic atrophy progress or remains constant, whether cognition can be improved after CEA and how the thalamic volume correlates with cognitive recovery. Third, some ACS patients in our study received lipidlowering management that may have subtle effects on 
subcortical nuclei morphology. ${ }^{33}$ Future studies should include medication-naive subjects to avoid this possible bias. Finally, although we accounted for the effect of small vessel disease by evaluating the history of stroke, present of intracranial artery stenosis and the degree of WMH, we did not exclude other possible relevant markers such as microbleeds.

\section{Conclusion}

This study demonstrated for the first time the significant alterations in subcortical nuclei and subregional volumes of these nuclei in ACS patients. Our findings indicated that patients with ACS have an increased risk of cognitive impairment and thalamic atrophy, especially in the medial dorsal nucleus of the thalamus. Furthermore, these structural changes were associated with impaired performance on tests of executive functioning. At the follow-up after CEA, cognitive improvement or recovery was observed 6 months after revascularization.

\section{Data Sharing Statement}

Data are available upon request.

\section{Ethics Approval and Informed Consent}

The study was approved by the Ethics Committee of the Drum Tower Hospital Affiliated to Nanjing University Medical School and was following the Declaration of Helsinki. All participants volunteered to participate in this study and written informed consents were obtained.

\section{Consent for Publication}

All authors have read and approved the submitted manuscript and were aware that if accepted the journal will have all rights regarding the publication.

\section{Acknowledgments}

The abstract of this paper was presented at the Advances in Alzheimer's and Parkinson's Therapies Conference (AAT-AD/PD 2020) as an e-poster presentation form with interim findings. The poster's abstract was published in "ePOSTER" section in website:https://cslide.ctimeeting tech.com/aat20/attendee/eposter/poster/141?s=pn.

\section{Funding}

This study was supported by the National Natural Science Foundation of China (grant number 81370387 and
81720108022). Zuzana Nedelska was supported by the International Brain Research Organization and International Society for Neurochemistry (IBRO-ISN) return home fellowship 2018. The funders had no role in the study design, data collection and analysis, decision to publish, or preparation of the manuscript.

\section{Disclosure}

The authors report no potential conflicts of interest in this work.

\section{References}

1. Touze E. Natural history of asymptomatic carotid artery stenosis. Rev Neurol (Paris). 2008;164(10):793-800. doi:10.1016/j.neurol.2008.07. 005

2. de la Torre JC. Vascular risk factor detection and control may prevent Alzheimer's disease. Ageing Res Rev. 2010;9(3):218-225. doi:10.10 16/j.arr.2010.04.002

3. Silvestrini M, Viticchi G, Falsetti L, et al. The role of carotid atherosclerosis in Alzheimer's disease progression. J Alzheimers Dis. 2011;25(4):719-726. doi:10.3233/JAD-2011-101968

4. Livingston G, Sommerlad A, Orgeta V, et al. Dementia prevention, intervention, and care. Lancet. 2017;390(10113):2673-2734. doi:10. 1016/S0140-6736(17)31363-6

5. Jack CR, Shiung MM, Weigand SD, et al. Brain atrophy rates predict subsequent clinical conversion in normal elderly and amnestic MCI. Neurology. 2005;65(8):1227-1231. doi:10.1212/01.wnl.0000180958. 22678.91

6. Alhusaini S, Karama S, Nguyen TV, et al. Association between carotid atheroma and cerebral cortex structure at age 73 years. Ann Neurol. 2018;84(4):576-587.

7. Avelar WM, D'Abreu A, Coan AC, et al. Asymptomatic carotid stenosis is associated with gray and white matter damage. Int J Stroke. 2015;10(8):1197-1203. doi:10.1111/ijs.12574

8. Hartkamp NS, Bokkers RP, van der Worp HB, et al. Distribution of cerebral blood flow in the caudate nucleus, lentiform nucleus and thalamus in patients with carotid artery stenosis. Eur Radiol. 2011;21 (4):875-881. doi:10.1007/s00330-010-1952-y

9. Hartkamp NS, Bokkers RP, van Osch MJ, et al. Cerebrovascular reactivity in the caudate nucleus, lentiform nucleus and thalamus in patients with carotid artery disease. J Neuroradiol. 2017;44 (2):143-150. doi:10.1016/j.neurad.2016.07.003

10. Li H, Jia J, Yang Z, Moreau N. Mini-mental state examination in elderly chinese: a population-based normative study. J Alzheimers Dis. 2016;53(2):487-496. doi:10.3233/JAD-160119

11. Fazekas F, Chawluk JB, Alavi A, et al. MR signal abnormalities at $1.5 \mathrm{~T}$ in Alzheimer's dementia and normal aging. AJR Am J Roentgenol. 1987;149(2):351-356. doi:10.2214/ajr.149.2.351

12. Jenkinson M, Beckmann CF, Behrens TE, et al. Fsl. Neuroimage. 2012;62(2):782-790.

13. Iglesias JE, Insausti R, Lerma-Usabiaga G, et al. A probabilistic atlas of the human thalamic nuclei combining ex vivo MRI and histology. Neuroimage. 2018;183:314-326. doi:10.1016/j.neuroimage.2018.08.012

14. Brott TG, Halperin JL, Abbara S, et al. Guideline on the management of patients with extracranial carotid and vertebral artery disease: executive summary. Circulation. 2011;124(4):489-532. doi:10.1161/ CIR.0b013e31820d8d78

15. Jack CR, Twomey CK, Zinsmeister AR, et al. Anterior temporal lobes and hippocampal formations: normative volumetric measurements from MR images in young adults. Radiology. 1989;172 (2):549-554. doi:10.1148/radiology.172.2.2748838 
16. Jack CR, Petersen RC, O’Brien PC, et al. MR-based hippocampal volumetry in the diagnosis of Alzheimer's disease. Neurology. 1992;42(1):183-188. doi:10.1212/WNL.42.1.183

17. Winkler AM, Ridgway GR, Webster MA, et al. Permutation inference for the general linear model. Neuroimage. 2014;92:381-397. doi:10.1016/j.neuroimage.2014.01.060

18. Hayes AF, Rockwood NJ. Regression-based statistical mediation and moderation analysis in clinical research: observations, recommendations, and implementation. Behav Res Ther. 2017;98:39-57. doi:10. 1016/j.brat.2016.11.001

19. Landgraff NC, Whitney SL, Rubinstein EN, et al. Cognitive and physical performance in patients with asymptomatic carotid artery disease. J Neurol. 2010;257(6):982-991. doi:10.1007/s00415-0095449-z

20. Chang XL, Zhou HQ, Lei CY, et al. Association between asymptomatic carotid stenosis and cognitive function: a systematic review. Neurosci Biobehav Rev. 2013;37(8):1493-1499. doi:10.1016/j.neubiorev.2013. 05.011

21. Sherman SM, Guillery RW. Functional Connections of Cortical Areas: A New View from the Thalamus. Cambridge, Mass: MIT Press; 2013.

22. Behrens TE, Johansen-Berg H, Woolrich MW, et al. Non-invasive mapping of connections between human thalamus and cortex using diffusion imaging. Nat Neurosci. 2003;6(7):750-757. doi:10.1038/ nn1075

23. Mitchell AS. The mediodorsal thalamus as a higher order thalamic relay nucleus important for learning and decision-making. Neurosci Biobehav Rev. 2015;54:76-88. doi:10.1016/j.neubiorev.2015.03.001

24. Khera AV, Cuchel M, de la Llera-moya M, et al. Cholesterol efflux capacity, high-density lipoprotein function, and atherosclerosis. N Engl J Med. 2011;364(2):127-135. doi:10.1056/NEJMoa1001689

25. Raitakari OT, Makinen VP, McQueen MJ, et al. Computationally estimated apolipoproteins B and A1 in predicting cardiovascular risk. Atherosclerosis. 2013;226(1):245-251. doi:10.1016/j.atherosclerosis. 2012.10.049
26. Farr SA, Yamada KA, Butterfield DA, et al. Obesity and hypertriglyceridemia produce cognitive impairment. Endocrinology. 2008;149 (5):2628-2636. doi:10.1210/en.2007-1722

27. Wagenknecht N, Becker B, Scheld M, et al. Thalamus degeneration and inflammation in two distinct multiple sclerosis animal models. J Mol Neurosci. 2016;60(1):102-114.

28. Hogberg D, Bjorck M, Mani K, et al. Five year outcomes in men screened for carotid artery stenosis at 65 years of age: a population based cohort study. Eur J Vasc Endovasc Surg. 2019;57(6):759-766. doi:10.1016/j.ejvs.2019.02.005

29. Altinbas A, van Zandvoort MJ, van den Berg E, et al. Cognition after carotid endarterectomy or stenting: a randomized comparison. Neurology. 2011;77(11):1084-1090. doi:10.1212/WNL.0b013e318 $22 \mathrm{e} 55 \mathrm{~b} 9$

30. Wilson DA, Mocco J, D'Ambrosio AL, et al. Post-carotid endarterectomy neurocognitive decline is associated with cerebral blood flow asymmetry on post-operative magnetic resonance perfusion brain scans. Neurol Res. 2008;30(3):302-306. doi:10.1179/016164107X23 0540

31. Wolf O, Heider P, Heinz M, et al. Microembolic signals detected by transcranial Doppler sonography during carotid endarterectomy and correlation with serial diffusion-weighted imaging. Stroke. 2004;35 (11):e373-5. doi:10.1161/01.STR.0000143184.69343.ec

32. O'Leary DH, Polak JF, Kronmal RA, et al. Distribution and correlates of sonographically detected carotid artery disease in the Cardiovascular Health Study. The CHS Collaborative Research Group. Stroke. 1992;23(12):1752-1760. doi:10.1161/01.STR.23.12. 1752

33. Corraliza-Gomez M, Sanchez D, Ganfornina MD. Lipid-binding proteins in brain health and disease. Front Neurol. 2019;10:1152. doi:10.3389/fneur.2019.01152
Clinical Interventions in Aging

\section{Publish your work in this journal}

Clinical Interventions in Aging is an international, peer-reviewed journal focusing on evidence-based reports on the value or lack thereof of treatments intended to prevent or delay the onset of maladaptive correlates of aging in human beings. This journal is indexed on PubMed Central, MedLine, CAS, Scopus and the Elsevier

\section{Dovepress}

Bibliographic databases. The manuscript management system is completely online and includes a very quick and fair peer-review system, which is all easy to use. Visit http://www.dovepress.com/ testimonials.php to read real quotes from published authors. 